\title{
Some Improvement upon Algorithm IrrCharSer
}

\author{
Fangjian Huang \\ School of Energy Science and Engeneering \\ University of Electronic Science and Technology of China \\ ChengDu, China, 611731 \\ huangfangjian@hotmail.com
}

\begin{abstract}
Li presents the U-set to improve the algorithm CharSer that can compute a characteristic series from any given polynomial set. We modify the definition of $U$-set and present a new version of the algorithm IrrCharSer. Examples are given to show that the improvement can avoid the redundant branches of the decomposition tree.
\end{abstract}

Keywords -- polynomial set, U-set,zero decomposition, algorithm IrrCharSer

\section{INTRODUCTION}

The research on polynomial system is used in many modern science branches such as: algebraic geometry, automatic theorem discovery, proof and reasoning, isolation of solution of polynomial system, inequation proof, computer algebra, computer vision, automatic control system. Initial set is one of the most fundamental definition in this area. Based on initial set, $\mathrm{Wu}$ [4-7] presents some algorithms to decompose the polynomial set into ascending sets and other sets. Li $[1,2]$ modifies the algorithm CharSer using Uset instead of initials and gets some wonderful improvements. We'll modify the definition of U-set and make some improvement on algorithm IrrCharSer which decompose the polynomial set into irreducible ascending sets.

In section I, we introduce some preliminaries of polynomial theory, improve the definition of U-set and give the algorithm of the modified U-set. The main results are put forward in section II, in which the algorithm IrrCharSer is optimized. Examples are given in section III to show that the advantages to the original algorithms.

Let $K$ be a field with characteristic $0, K\left[x_{1}, \cdots, x_{n}\right]$ ( $K[X]$ for short) the ring of polynomials in the variables $\left(x_{1}, \cdots, x_{n}\right)$ ( $X$ for short) with coefficients in $K$ and $\widetilde{K}$ be the extension field of $K$. Let $T$ be a triangular set in $K[X], p \in K[X] \backslash K$, then $\operatorname{res}(p, T)$ stands for the resultant of $p$ with respect to $T$.

For any n-dimension polynomial set $P$ in $K[X]$, and any polynomial system, $[P, Q]$, the set of all zeros in $\widetilde{K}^{n}$ are denoted respectively as the following:

$$
\begin{aligned}
& \operatorname{Zero}(P)=\left\{z \in \widetilde{K}^{n}: p(z)=0, \forall p \in P\right\} \\
& \operatorname{Zero}(P / Q)=\left\{z \in \widetilde{K}^{n}: p(z)=0, q(z) \neq 0, \forall p \in P, q \in Q\right\}
\end{aligned}
$$

For any triangular set $T$ in $K[X], \mathrm{Li}[1,2]$ presents the definition of $U_{T}$. We modify it as the following:
Definition 1.1 The U-set for triangular set $T$ is

$$
\begin{aligned}
& U_{T}=\{c: \operatorname{res}(c, T)=0, c \in \operatorname{ini}(T)\} \bigcup \\
& \quad\left\{r: \text { one } r \in R_{f}, \operatorname{res}(\operatorname{ini}(f), T) \neq 0, R_{f} \cap K=\varnothing, f \in T\right\}
\end{aligned}
$$

in which

$$
R_{f}=\left\{\operatorname{res}(c, T) \neq 0: c \in C_{f}\right\} \quad \forall f \in T
$$

$C_{f}$ is the set of coefficients of $f$ and $\operatorname{ini}(T)$ is the set of initials of $T$.

It's easy to see that all the propositions in $[1,2]$ hold for the new definition of U-set. And the following algorithm is presented to compute the U-set.

Algorithm (CompA): $U_{T} \leftarrow \operatorname{Comp} A(T)$. This algorithm computers the U-set of any given triangular set $T \in K[X]$.

C1.Let $U \leftarrow \varnothing, T^{*} \leftarrow T$;

C2. while $T^{*} \neq \varnothing$ do:

C2.1. Choose any one polynomial $\mathrm{f}$ from $T^{*}$, and set $T^{*} \leftarrow T^{*} \backslash\{f\}$

C2.2. Let $r \leftarrow \operatorname{res}(\operatorname{ini}(f), T)$;

C2.2.1. if $r=0$ then $U \leftarrow U \cup\{\operatorname{ini}(f)\}$;

C2.2.2. if $r \neq 0$ and $C_{f} \cap K=\varnothing$ then let

$$
R_{f}=\left\{\operatorname{res}(c, T): \forall c \in C_{f}\right\} \backslash\{0\} ;
$$

C2.2.3. if $R_{f} \cap K=\varnothing$ then choose any one element $R$ from $R_{f}$;

C2.2.4. Set $U \leftarrow U \cup\{R\}$;

C3. Let $U_{T} \leftarrow U$.

\section{MAIN RESULTS}

The irreducible decomposition is one of the most classical algorithm to decompose the solution sets of polynomial set. It's presented by $\mathrm{Wu}$ [7]. One can find the details from some text book such as [3]. Using U-set to take the place of initials, we get the following algorithm IrrCharSerA according to the IrrCharSer algorithm in [3].

Algorithm (IrrCharSerA): $\Psi \leftarrow \operatorname{IrrCharSer} A(P)$. Given a nonempty polynomial set $P \subset K[X]$, this algorithm decomposes it into irreducible characteristic ascending sets with the same solution set.

IA1. Let $\Phi \leftarrow\{P\}, \Psi \leftarrow \varnothing$;

IA2. While $\Phi \neq \varnothing$ do:

IA2.1. Choose any one element $F$ from $\Phi$, and let $\Phi \leftarrow \Phi \backslash\{F\}$; 
IA2.2. Compute $C \leftarrow \operatorname{CharSet}(F)$;

IA2.3. If $C$ is noncontradictory then

IA2.3.1. $[k, D, G] \leftarrow \operatorname{Factor}(C)$;

IA2.3.2. If $k=0$ then

IA2.3.2.1. Compute $U_{C} \leftarrow \operatorname{CompA}(C)$.

IA2.3.2.2. Let $\Psi \leftarrow \Psi \cup\{C\}$, and

$$
\Phi \leftarrow \Phi \bigcup \bigcup_{I \in U_{C}}\{F \bigcup C \bigcup\{I\}\}
$$

IA2.3.3. If $k \neq 0$ then let

$$
U_{C^{(k)}} \leftarrow \operatorname{CompA}\left(C^{(k)}\right)
$$

and

$$
\Phi \leftarrow \Phi \cup \underset{T \in U_{C}(k)}{\bigcup}\{F \cup C \cup\{T\}\} \cup \bigcup_{g \in G}\{F \cup C \cup\{g\}\}
$$

We can get that

$\operatorname{Zero}(P)=\bigcup_{C \in \Psi} \operatorname{Zero}\left(C / U_{C}\right), \quad \Psi=\operatorname{IrrCharSerA}(P)$

Because we just use the U-set instead of initial set, the termination and correctness of this algorithm are easily checked from the definition of U-set.

Here, the function Factor $(C)$ [3] determines whether the triangular set is irreducible or not and factor the reducible elementary.

Algorithm (Factor): $[k, D, F] \leftarrow$ Factor $(C)$. For any fine triangular polynomial set $C \subset K[X]$, this algorithm computes an integer $k$, a polynomial $D$ and a polynomial set $F \subset K[X]$, such that $|0 \leq k \leq| C \mid$, and

I. If $k=0$, then $C$ is irreducible;

II. If $k=1$, then $C$ is reducible, $|F|>1 . C_{1}$, the first polynomial of class $p_{1}$ in $C$, can be factored into $C_{1}=\prod_{f \in F} f$ over field $K_{0}=K\left(x_{1}, \cdots, x_{p_{1}-1}\right)$. And every $f \in F \subset K_{0}\left[x_{p_{1}}\right]$ is irreducible in $K_{0}$;

III. If $k>1$, then $C$ is reducible and $C^{\{k-1\}}$ is irreducible, $|F|>1 . C_{k}$, the $k$ th polynomial in $C$ has a factorization $D C_{k} \doteq \prod_{f \in F} f$ over the field $K_{k-1}$ which is the extension field of $K$ with the adjoining triangular set $C^{\{k-1\}}$. Every $f \in F \subset K_{k-1}\left[x_{p_{k}}\right]$ is irreducible over $K_{k-1}$.

In III., we extend the base field $K$ into the extension field $K_{k-1}$ in a specific way : $K_{k-1}=K\left(x_{1}, \cdots, x_{p_{k}-1}\right)$. For any $1 \leq j \leq k-1, x_{p_{j}}$, the leading variable of $C_{j}$, is considered as an algebraic element with adjoining polynomial $C_{j}$ while the other variables are adjoined as transcendental ones.

\section{APPLICATIONS}

In this section, we will present two examples to compare the our algorithm with IrrCharSer in maple package epsilon.

Example 1. Let $P=\left\{p_{1}, p_{2}, p_{3}\right\}$, and

$$
\begin{aligned}
& p_{1}=-x_{1}-x_{3}+x_{4}^{2}, \\
& p_{2}=x_{4}+x_{1} x_{4}-x_{2} x_{4}-x_{4}^{2}, \\
& p_{3}=x_{1}^{2}-x_{1} x_{4}-x_{2} x_{4}
\end{aligned}
$$

with the variable ordering $x_{1} \prec x_{2} \prec x_{3} \prec x_{4}$ in the polynomial ring $\mathrm{Q}\left[x_{1}, x_{2}, x_{3}, x_{4}\right]$ with coefficient field $\mathrm{Q}$.

With algorithm IrrCharSerA, We get the decomposition of its solution set as the following:

$$
\operatorname{Zero}(\mathrm{P})=\operatorname{Zero}\left(\mathrm{C}_{1}\right) \cup \operatorname{Zero}\left(\mathrm{C}_{2}\right) \cup \operatorname{Zero}\left(\mathrm{C}_{3} /\left\{x_{1}\right\}\right)
$$
in which

$$
\begin{aligned}
& C_{1}=\left[\begin{array}{l}
x_{1} \\
x_{3} \\
x_{4}
\end{array}\right] C_{2}=\left[\begin{array}{l}
x_{1} \\
x_{2} \\
x_{3}-1 \\
-1+x_{4}
\end{array}\right] \mathrm{C}_{3}=\left[\begin{array}{l}
-x_{2}^{2}+x_{2}+x_{1} \\
C_{32} \\
\left(-x_{1}-x_{2}\right) x_{4}+x_{1}^{2}
\end{array}\right] \\
& C_{32}=\left(-x_{1}^{2}-x_{1}-2 x_{1} x_{2}-x_{2}\right) x_{3}+x_{1}^{4}-x_{1}^{3}-x_{1}^{2}-2 x_{1}^{2} x_{2}-x_{1} x_{2}
\end{aligned}
$$

Remark 1 Using the Maple software package epsilon, we can get the following decomposition:

$\operatorname{Zero}(\mathrm{P})=\operatorname{Zero}\left(\mathrm{C}_{1}\right) \cup \operatorname{Zero}\left(\mathrm{C}_{2}\right)$

$$
\cup \operatorname{Zero}\left(\mathrm{C}_{3} /\left\{\left(-x_{1}-x_{2}\right) x_{1}^{2},-x_{1}^{2}-2 x_{1} x_{2}-x_{1}-x_{2}\right\}\right)
$$

in which $\mathrm{C}_{1}, \mathrm{C}_{2}, \mathrm{C}_{1}$ are the same as above. The difference between the two decompositions is the U-set and the initials of $\mathrm{C}_{3}$. Using the algorithm CompA, one can compute the $\mathrm{U}$ set of $C_{3}$

$$
\mathrm{U}_{\mathrm{C}_{3}}=\left\{x_{1}\right\}
$$

and

$$
\operatorname{ini}\left(\mathrm{C}_{3}\right)=\left\{\left(-x_{1}-x_{2}\right) x_{1}^{2},-x_{1}^{2}-2 x_{1} x_{2}-x_{1}-x_{2}\right\} .
$$

Cause Zero $\left(\mathrm{C}_{3} \cup\left\{x_{1}+x_{2}\right\}\right)=\left\{x_{1}=0, x_{2}=0\right\}$, we have

$$
\operatorname{Zero}\left(\mathrm{C}_{3} /\left\{x_{1}\right\}\right)
$$

$=\operatorname{Zero}\left(\mathrm{C}_{3} /\left\{\left(-x_{1}-x_{2}\right) x_{1}^{2},-x_{1}^{2}-2 x_{1} x_{2}-x_{1}-x_{2}\right\}\right)$

So, we can find that the decomposition using U-set is more concise than the one using the initials.

Example 2. Given a polynomial set:

$$
F=\left\{f_{1}, f_{2}, f_{3}\right\} \subset Q\left[x_{1}, x_{2}, x_{3}, x_{4}\right]
$$

in which

$$
\begin{aligned}
& f_{1}=x_{1}-x_{2}+x_{3}+x_{2}^{2}, \\
& f_{2}=-x_{2}+x_{1}^{2}+x_{2}^{2}-x_{3} x_{4}, \\
& f_{3}=x_{1}+x_{1}^{2}+x_{1} x_{4}+x_{4}^{2}
\end{aligned}
$$

The IrrCharSerA returns the following decomposition of solution set with the variables ordering $x_{1} \prec x_{2} \prec x_{3} \prec x_{4}$ : $\operatorname{Zero}(\mathrm{F})=\operatorname{Zero}\left(\mathrm{C}_{1}\right) \cup \operatorname{Zero}\left(\mathrm{C}_{2}\right)$

$$
\cup \operatorname{Zero}\left(\mathrm{C}_{3}\right) \cup \operatorname{Zero}\left(\mathrm{C}_{4} /\left\{x_{1}, x_{1}+1, x_{1}-1\right\}\right)
$$

in which 
$\mathrm{C}_{1}=\left[\begin{array}{l}x_{1} \\ x_{2} \\ x_{3} \\ x_{4}\end{array}\right] \mathrm{C}_{2}=\left[\begin{array}{l}x_{1} \\ x_{2}-1 \\ x_{3} \\ x_{4}\end{array}\right] \mathrm{C}_{3}=\left[\begin{array}{l}x_{1}-1 \\ x_{2}^{2}-x_{2}+1 \\ x_{3} \\ x_{4}^{2}+x_{4}+2\end{array}\right] \mathrm{C}_{4}=\left[\begin{array}{l}C_{41} \\ x_{3}+x_{2}^{2}-x_{2}+x_{1} \\ \left(x_{1}-x_{2}+x_{2}^{2}\right) x_{4}-x_{2}+x_{1}^{2}+x_{2}^{2}\end{array}\right]$ $C_{41}=\left(x_{1}^{2}+1\right)\left(x_{2}^{4}-2 x_{2}^{3}\right)+\left(4 x_{1}^{2}+x_{1}^{3}+1\right) x_{2}^{2}+\left(-3 x_{1}^{2}-x_{1}^{3}\right) x_{2}+x_{1}^{3}+x_{1}^{4}$

Remark 2 Using the ics function in epsilon package, we get that

$\operatorname{Zero}(F)=\operatorname{Zero}\left(\mathrm{C}_{1}\right) \cup \operatorname{Zero}\left(\mathrm{C}_{2}\right) \cup \operatorname{Zero}\left(\mathrm{C}_{3}\right)$

$\cup \operatorname{Zero}\left(\mathrm{C}_{4} /\left\{x_{1}-x_{2}+x_{2}^{2}, x_{1}^{2}+1\right\}\right) \cup \operatorname{Zero}\left(\mathrm{C}_{5} /\left\{x_{1}+3, x_{1}\right\}\right)$

in which $\mathrm{C}_{1}, \mathrm{C}_{2}, \mathrm{C}_{3}, \mathrm{C}_{4}$ are the same as above while

$$
\mathrm{C}_{5}=\left[\begin{array}{l}
x_{1}^{2}+1 \\
\left(x_{1}+3\right) x_{2}^{2}+\left(-3-x_{1}\right) x_{2}+x_{1}-1 \\
\left(x_{1}+3\right) x_{3}+2 x_{1} \\
x_{1} x_{4}-x_{1}-1
\end{array}\right]
$$

It's easy to find that:

$\operatorname{Zero}\left(\mathrm{C}_{5} /\left\{x_{1}+3, x_{1}\right\}\right) \subset \operatorname{Zero}\left(\mathrm{C}_{4} /\left\{x_{1}, x_{1}+1, x_{1}-1\right\}\right)$.

So we can see that U-set is useful to avoid the redundant branches of the decomposition tree.

Example 3. Let polynomial set $H=\left\{h_{1}, h_{2}, h_{3}\right\}$ be in the polynomial ring $Q\left[x_{1}, x_{2}, x_{3}, x_{4}\right]$ with coefficient field $Q$ and the variable ordering $x_{1} \prec x_{2} \prec x_{3} \prec x_{4}$.

$$
\begin{aligned}
& h_{1}=-\left(x_{1}+1\right) x_{4}+x_{3}^{2}-x_{1} x_{3}-x_{1}+1, \\
& h_{2}=-x_{3} x_{1}+x_{2}^{2}-x_{2} x_{1}+x_{1}^{2}-x_{1}+1, \\
& h_{3}=\left(x_{3}-x_{1}\right) x_{4}-x_{3} x_{2}-x_{2}^{2}
\end{aligned}
$$

Using the algorithm IrrCharSerA, one can decompose the polynomial set $H$ into the series of irreducible ascending sets $\left\{C_{1}, C_{2}, C_{3}, C_{4}\right\}$ in which

$$
\begin{gathered}
C_{1}=\left[\begin{array}{c}
c_{11} \\
-x_{3} x_{1}+x_{2}^{2}-x_{2} x_{1}+x_{1}^{2}-x_{1}+1 \\
c_{13}
\end{array}\right] \\
C_{2}=\left[\begin{array}{c}
x_{1}+1 \\
x_{2}^{4}+2 x_{2}^{3}+6 x_{2}^{2}+5 x_{2}+8 \\
x_{3}+x_{2}^{2}+x_{2}+3 \\
\left(x_{2}^{2}+x_{2}+2\right) x_{4}-x_{2}^{3}-3 x_{2}
\end{array}\right] \\
C_{3}=\left[\begin{array}{c}
x_{1} \\
x_{2}^{2}+1 \\
x_{3}^{2}-x_{3} x_{2}-x_{2} \\
x_{4}-x_{3} x_{2}-x_{2}-1
\end{array}\right] C_{4}=\left[\begin{array}{c}
x_{2}^{2}+1 \\
x_{3}+x_{2} \\
x_{4}
\end{array}\right]
\end{gathered}
$$

$$
\begin{aligned}
c_{11}= & -x_{2}^{6}+3 x_{1} x_{2}^{5}+\left(-4 x_{1}^{2}+3 x_{1}-3\right) x_{2}^{4}+\left(4 x_{1}^{3}-5 x_{1}^{2}\right. \\
& \left.+6 x_{1}\right) x_{2}^{3}+\left(-x_{1}^{4}+6 x_{1}^{3}-9 x_{1}^{2}+6 x_{1}-3\right) x_{2}^{2}+\left(x_{1}^{5}\right. \\
& \left.-3 x_{1}^{4}+6 x_{1}^{3}-5 x_{1}^{2}+3 x_{1}\right) x_{2}-2 x_{1}^{4}+5 x_{1}^{3}-5 x_{1}^{2} \\
& +3 x_{1}-1 \\
c_{13}= & \left(x_{1}^{3}+x_{1}^{2}\right) x_{4}-x_{2}^{4}+2 x_{2}^{3} x_{1}+\left(-2 x_{1}^{2}+2 x_{1}-2\right) x_{2}^{2} \\
& \left(x_{1}^{2}-2 x_{1}+2\right) x_{2} x_{1}+2 x_{1}^{3}-3 x_{1}^{2}+2 x_{1}-1
\end{aligned}
$$

And the zero set relationship is Zero $(H)=$ Zero $\left(C_{1} /\left\{x_{1}+1, x_{1}\right\}\right)$

$$
\cup \operatorname{Zero}\left(C_{2}\right) \cup \operatorname{Zero}\left(C_{3}\right) \cup \operatorname{Zero}\left(C_{4}\right)
$$

Remark 3 Using the function ics in epsilon package, we can get that

Zero $(H)=$ Zero $\left(C_{1} /\left\{x_{1}+1, x_{1}\right\}\right) \cup$ Zero $\left(C_{2} /\left\{x_{2}^{2}+x_{2}+2\right\}\right)$

\section{$\operatorname{Zero}\left(C_{3}\right) \cup \operatorname{Zero}\left(C_{4}\right)$}

in which $C_{1}, C_{2}, C_{3}, C_{4}$ are the same as above. It's easy to see that

$$
\operatorname{Zero}\left(C_{1}\right) \subseteq \operatorname{Zero}(H) \text { and } \operatorname{Zero}\left(C_{2}\right) \subseteq \operatorname{Zero}(H)
$$

Example 4. Given a polynomial set $P=\left\{p_{1}, p_{2}, p_{3}\right\}$ in the polynomial ring $Q\left[x_{1}, x_{2}, x_{3}, x_{4}\right]$, and

$$
\begin{aligned}
& p_{1}=-2 x_{4} x_{1}-x_{3}^{2}-2 x_{3} x_{1}, \\
& p_{2}=2 x_{4}^{2}+2 x_{4}+x_{1}, \\
& p_{3}=2\left(1+x_{2}\right) x_{3}+x_{2}
\end{aligned}
$$

one can decompose it into

$$
\operatorname{Zero}(P)=\operatorname{Zero}\left(C_{1} /\left\{x_{1}\right\}\right) \cup \operatorname{Zero}\left(C_{2}\right) \cup \operatorname{Zero}\left(C_{3}\right)
$$
using the algorithm IrrCharSerA, in which

$$
\begin{aligned}
C_{1}= & {\left[\begin{array}{c}
c_{11} \\
2\left(1+x_{2}\right) x_{3}+x_{2} \\
c_{13}
\end{array}\right] C_{2}=\left[\begin{array}{c}
x_{1} \\
x_{2} \\
x_{3} \\
1+x_{4}
\end{array}\right] C_{3}=\left[\begin{array}{c}
x_{1} \\
x_{2} \\
x_{3} \\
x_{4}
\end{array}\right] } \\
c_{11}= & \left(32 x_{1}^{3}+48 x_{1}^{2}-16 x_{1}+1\right) x_{2}^{4}+8\left(16 x_{1}^{2}+16 x_{1}-3\right) x_{2}^{3} x_{1} \\
& 8\left(24 x_{1}^{2}+14 x_{1}-1\right) x_{2}^{2} x_{1}+32 x_{1}^{2}\left(4 x_{1}+1\right) x_{2}+32 x_{1}^{3} \\
c_{13}= & 8\left(1+x_{2}\right)^{2} x_{4} x_{1}-x_{2}\left(x_{2}\left(4 x_{1}-1\right)+4 x_{1}\right)
\end{aligned}
$$

Remark 4.Using function ics, polynomial set $P$ in Example 4 can be decomposed into

$\operatorname{Zero}(P)=\operatorname{Zero}\left(C_{1} / I_{1}\right) \cup \operatorname{Zero}\left(C_{2}\right) \cup \operatorname{Zero}\left(C_{3}\right)$

$$
\cup \operatorname{Zero}\left(C_{4} / I_{4}\right) \cup \operatorname{Zero}\left(C_{5} / I_{5}\right)
$$

in which $C_{1}, C_{2}, C_{3}$ are as above and

$I_{1}=\left\{x_{2}+1,32 x_{1}^{3}+48 x_{1}^{2}-16 x_{1}+1, x_{1}\right\}$

$I_{4}=\left\{x_{2}+1,8 x_{1}-1, x_{1}\right\}, I_{5}=\left\{2 x_{1}-1, x_{1}\right\}$ 


$$
\begin{aligned}
& C_{4}=\left[\begin{array}{c}
32 x_{1}^{3}+48 x_{1}^{2}-16 x_{1}+1 \\
\left(16 x_{1}-2\right) x_{2}^{2}+2\left(14 x_{1}-1\right) x_{2}+12 x_{1}-1 \\
2\left(x_{2}+1\right) x_{3}+x_{2} \\
8 x_{4} x_{1}+12 x_{1}-1
\end{array}\right] \\
& C_{5}=\left[\begin{array}{c}
32 x_{1}^{3}+48 x_{1}^{2}-16 x_{1}+1 \\
2\left(2 x_{1}-1\right) x_{2}+4 x_{1}-1 \\
2 x_{3}+4 x_{1}-1 \\
8 x_{1} x_{4}-4 x_{1}+1
\end{array}\right]
\end{aligned}
$$

In fact, there exists

$$
\text { Zero }\left(C_{1} /\left\{x_{1}\right\}\right)=\text { Zero }\left(C_{1} / I_{1}\right) \cup \text { Zero }\left(C_{4} / I_{4}\right) \cup \text { Zero }\left(C_{5} / I_{5}\right)
$$

The two examples above show that the decomposition using $\mathrm{U}$-set is more concise than the one using the initials.

\section{CONCLUSION}

In this paper, we modify the definition of U-set and improve the algorithm IrrCharSer. The decomposition results of Example1,3 and 4 show that the modified algorithm can return the more concise result. The result of Example 2 show that it also can avoid the redundant branches of the decomposition tree. The U-set works more efficiently than initials. We'll modify the other algorithms with U-set in future.

\section{ACKNOWLEDGEMENT}

This work is partially supported by National Natural Science Foundation of China (61074189). The author would like to thank his colleagues for their advices and encouragement.

\section{REFERENCES}

[1] Li Y.-B. Some Notes upon When does equal sat? AISC[J], 2010, PP:89-100.

[2] Li Y.-B. Some properties of triangular sets and improvement upon algorithm charser. AISC[J], 2006, PP:224:260-266.

[3] Wang D.-M. Elimination methods. Springer[B], Wien New York, 2001.

[4] Wu W.-T. A mechanization method of geometry and its applications $\mathrm{i}$. distances, areas and volumes. J. Syst. Sci. Math. Sci.[J], 1986, 6:204216.

[5] Wu W.-T. On reducibility problem in mechanical theorem proving of elementary geometries. Chinese Quarterly J. of Math.[J], 1987, PP:2:1-19, also in MM-Res.Preprints, No.2[J], 1987, PP:18-36.

[6] Wu W.-T. On the decision problem and the mechanization of theorem-proving in elementary geometry. Science China[J],1977, PP:6:507-516

[7] Wu W.-T. On the generic zero and chow basis of an irreducible ascending set. Math. Mech. Res. Preprints, 1989, PP:4:1-21. 